\title{
Evaluation of XpertMTB/RIF performance for diagnosis of tuberculosis among HIV positive patients in northern Tanzania
}

\author{
EDSON W. MOLLEL ${ }^{1,2}$, JAFFU O. CHILONGOLA ${ }^{1,3^{*}}$, STELLAH G. MPAGAMA² and GIBSON S. KIBIKI ${ }^{3}$ \\ ${ }^{1}$ Kilimanjaro Christian Medical University College, P.O. Box 2240, Moshi, Tanzania \\ ${ }^{2}$ Kibong'oto Infectious Diseases Hospital, P.O. Box 12, Sanya Juu, Tanzania \\ ${ }^{3}$ Kilimanjaro Clinical Research Institute, P.O. Box 2236, Moshi, Tanzania
}

\begin{abstract}
Background: Diagnosis of tuberculosis (TB) in patients co-infected with HIV poses an important challenge because of the low sensitivity and specificity of microscopy, the standard diagnostic method. This study aimed to evaluate the performance of the XpertMTB/RIF tool for TB diagnosis among TB-HIV co-infected patients. Methods: This was a cross-sectional analytical study conducted at Kibong'oto Infectious Disease Hospital in northern Tanzania and involved 69 patients. The performance of the XpertMTB/RIF and microscopy using $L J$ culture were determined and compared. Demographic, clinical, laboratory and radiological data were collected.

Results: XpertMTB/RIF had a higher sensitivity (100\%), specificity (100\%), positive predictive value (79.2\%) and negative predictive value (98.9\%) as compared to microscopy. There was a strong correlation between XpertMTB/RIF, microscopy and Löwenstein-Jensen ( $L J)$ culture in terms of their sensitivity and specificity.

Conclusion: While using TB symptoms screening tool alone in HIV infected individuals may result into overtreatment, relying on microscopy alone has the potential of TB under-diagnosing, miss-diagnosing and delayed treatment. Our results show XpertMTB/RIF to be highly sensitive and specific to detect all culture positive TB cases among HIV patients. We recommend the adoption of XpertMTB/RIF as an early TB diagnosis tool among HIV patients for early detection of TB among HIV patients.
\end{abstract}

Keywords: tuberculosis, HIV/AIDS, XpertMTB/RIF, diagnosis, performance, Tanzania

\section{Introduction}

Tuberculosis (TB) remains among the leading global infectious diseases that are responsible for higher mortalities. The burden of this disease has been high globally for more than ten years. Recent estimates by the World Health Organization indicate that the incidence of TB among people living with HIV is 62,000 with mortality rate of 53 per 100,000 (WHO, 2015). There has been an increase in global incidence of TB by 40\% from 1990 to 2008 (World Health Organization, 2009). This increase in TB infection rate has been attributed to HIV epidemic (World Health Organization, 2009). Global TB data show that in 2014, there were approximately 9.6 million incidence of TB. In the same year, data indicate that there were an estimated 1.2 million new HIV-positive TB cases accounting for about $12 \%$ of all TB cases, three-quarters of which were from the African Region (World Health Organization, 2015). Although the prevalence of TB among people living with HIV/AIDS in Tanzania was previously reported to be $8.5 \%$ in 2008 (Ngowi et al., 2008), the prevalence is likely to be higher at present.

HIV infection is known to be associated with tuberculosis, a phenomenon that worsens the outcome of either of the co-infecting diseases, particularly so if diagnosis and treatment are delayed (Gandhi et al., 2010; Kwan \& Ernst, 2011). This fact calls for the need of prompt and accurate diagnosis of the infections and treatment initiated as early as possible. The use of microscopy alone in detection of TB positive individuals among HIV infected patients has frequently led to missing of substantial numbers of true TB positive cases in Tanzania (Swai et al., 2011) with consequences of providing Isoniazid Prophylactic Therapy (IPT) to inappropriate individuals who would otherwise deserve a full course of TB treatment. Together with other factors, misdiagnosis and mistreatment have the obvious

\footnotetext{
${ }^{*}$ Correspondence E-mail: jchilongola@kcri.ac.tz
} 
adverse consequences of development of isoniazid resistance mycobacteria (Balcells et al., 2006) and hence are likely to result into multi-drug resistant tuberculosis (MDR-TB).

The XpertMTB/RIF tool, endorsed by WHO in 2011 (WHO, 2013) has the potential to diagnose TB and MDR-TB earlier and more accurately than microscopy. It has been introduced in Tanzania programmatic TB diagnostic algorithm among HIV patients in 2011. We envisage that the adoption and use of new diagnostic tools with increased accuracy for TB diagnosis such as XpertMTB/RIF can be integrated in active TB case screening and significantly increase the success of TB control programmes which are virtually using microscopy as the principal diagnostic tool. However, the XpertMTB/RIF performance in active case detection among HIV positive TB patients has not been evaluated in Tanzania. The HIV infected patients tend to have atypical and/or asymptomatic TB leading to difficult and delayed diagnosis when using microscopy, and hence increased rates of morbidity and mortality (Aderaye et al., 2004). In this study, we evaluated the performance and effectiveness of XpertMTB/RIF, compared to microscopy, in TB detection among HIV positive patients using Lowenstein-Jensen culture as a gold standard.

\section{Materials and Methods}

\section{Study site and design}

This was a cross-sectional analytical study conducted at Kibong'oto Infectious Diseases Hospital (KIDH) from September 2014 to March 2015. Consecutive sampling technique was used whereby all patients seen at the hospital Care and Treatment Centre (CTC) during data collection period were included in the study if they met all inclusion criteria. The inclusion criteria were: consenting to participate in the study, being HIV positive and anti-retroviral (ARV) naive and being able to produce enough sputum for investigation. All patients who had been on isoniazid prophylactic therapy (IPT) or anti-TB therapy for the past 18 months from the time of study commencement were excluded.

\section{Data collection}

The study enrolled 78 patients who were HIV positive and ARVs naïve, who were above 15 years of age and attending at the KIDH HIV clinic for care and treatment. Patients who fulfilled the eligibility criteria were included. Patients' demographic data were collected using a specially designed data capturing form. The World Health Organization tuberculosis symptoms screening tool was used to look for any cardinal TB symptoms. Subsequently patients were instructed on how to collect the sputa (>5mls) which was portioned for microscopy (ZN), Löwenstein-Jensen (LJ) culture and XpertMTB/RIF investigations. Nine patients were excluded because they were either not able to produce enough sputum, or their sputa were of low quality leading to inability to properly do one or both of the sputum investigations (microscopy, XpertMTB/RIF, LJ culture).

Sputum smear slides for microscopy were prepared by flooding slides with Auramine stain for about 20 minutes. Then slides were rinsed with tap water and a decolorizing solution (acid-alcohol) was poured on the slides and left for about 2 minutes. Slides were rinsed again with excess water and flooded with potassium permanganate solution for a minute. After a final rinsing, slides were left to air-dry. Dry slides were examined by microscopy. Sputum for XpertMTB/RIF investigation was mixed with the XpertMTB/RIF buffer solution, and shaken vigorously 20 times. The mixture was then incubated at room temperature for about 5 minutes. It was then shaken again 20 times and left to incubate for another 10 minutes. Afterwards, diluted specimen samples were pipetted into the appropriate cartridge of the XpertMTB/RIF for TB diagnosis. The automatically computer generated results were read.

Sputum portion for LJ (Lowenstein-Jensen) culture was initially decontaminated with $4 \%$ of sodium hydroxide followed by addition of sufficient amount of distilled water to achieve a volume of 
$50 \mathrm{ml}$ in a centrifuge tube. The mixture was then centrifuged at $1,800 \mathrm{X} g$ for 30 minutes. Sediments were inoculated on the $L J$ media slant using a sterile Pasteur pipette. The tubes were left overnight and then put back in an up-right position with loosened caps for 72 hours after when tube caps were tightened. Results were read weekly from the inoculation day for eight weeks. All laboratory procedures were performed at Kibong'oto Hospital Laboratory. Regardless of their sputum results, all study participants had chest radiography, provided blood for full blood picture and CD4 count (The later only collected if it was not done and/or documented in the patient's hospital file within the previous month). Samples and data collection from the participating patients were done at a single encounter with the patient.

\section{Data analysis}

Data were analysed by using the STATA software where associations were determined by using Chisquare test and Mann-Whitney test. Sensitivity, specificity, positive predictive values and negative predictive values were calculated by using an R package called BDPV. Cut off value for alpha ( $\alpha$ ) was 0.05 .

\section{Ethical considerations}

Ethical clearance was sought from Kilimanjaro Christian Medical University College Research and Ethics Committee (Certificate \# 726). Permission from KIDH Management was obtained. All participants consented to participate in the study voluntarily. Patients' confidentiality and privacy were strictly observed.

\section{Results}

A total of 78 patients were involved and 69 of them were analysed. Thirty-eight, (55\%) of those analysed were females, and $55(80 \%)$ were below 50 years of age (Table 1 ). The mean and median age of the study population was 41.8 and 42 years, respectively. Eighteen (26\%) patients had used some medications including antibiotics two weeks prior to enrolment. Fifty-seven (83\%) of these patients were on co-trimoxazole preventive therapy (CPT).

Table 1: Patients' characteristics distribution $(n=69)$

\begin{tabular}{llll}
\hline Variable & Category & N & Percent (\%) \\
\hline Age & Above 50 years & 14 & 20 \\
& Below 50 years & 55 & 80 \\
Sex & Male & 31 & 45 \\
Domicile & Female & 38 & 55 \\
& Within Siha District & 44 & 64 \\
Education & Outside Siha District & 25 & 36 \\
\multirow{2}{*}{ Occupation } & Primary & 64 & 93 \\
& Post Primary & 5 & 7 \\
Cigarette Smoking & Mining & 4 & 6 \\
Alcohol drinking & Others & 65 & 94 \\
& Yes & 9 & 13 \\
Previous antibiotic medication & No & 60 & 87 \\
Cotrimoxazole & Yes & 21 & 30 \\
& No & 48 & 70 \\
& Yes & 18 & 26 \\
\hline
\end{tabular}


All 9 patients diagnosed to have TB had at least one of the TB symptoms. Six (67\%) of those diagnosed with TB presented with cough though the difference was not statistically significant compared to those who had no cough. Six (0.09\%) of all patients presented with haemoptysis but only 2 (33.3\%) of them had a positive LJ culture. Night sweat, presence of consolidation and cavities in chest radiographs were associated with the increased chance of diagnosing TB with either XpertMTB/RIF or $L J$ culture, $p$-values are $0.022,<0.000$ and $<0.000$ respectively. Being symptomatic or asymptomatic for TB using the WHO symptom screening tool, was not associated with the diagnosis of TB by XpertMTB/RIF or culture LJ (Table 2). None of the study patients was found to have pleural effusion. Five (71.4\%) patients among those who had consolidation were diagnosed to have TB, and 5 (62.5\%) of the 8 patients with cavities had TB (Table 2 ).

Table 2: Association of TB features in HIV co-infection with XpertMTB/RIF results

\begin{tabular}{|c|c|c|c|c|c|}
\hline TB Feature & Symptoms & $\begin{array}{l}\text { Microscopy } \\
+ \text { ve }\end{array}$ & $\begin{array}{l}\text { L culture } \\
\text { +ve }\end{array}$ & $\begin{array}{l}\text { XpertMTB/RIF } \\
\text { +ve }\end{array}$ & $\mathrm{X}^{2} \mathrm{p}$ value \\
\hline \multirow[t]{2}{*}{ Cough } & $Y(36)$ & 4 & 6 & 6 & 0.351 \\
\hline & $N(33)$ & 2 & 3 & 3 & \\
\hline \multirow[t]{2}{*}{ Haemoptysis } & $Y(6)$ & 2 & 2 & 2 & 0.122 \\
\hline & $N(63)$ & 4 & 7 & 7 & \\
\hline \multirow[t]{2}{*}{ Fever } & $Y(21)$ & 2 & 4 & 4 & 0.327 \\
\hline & $N(48)$ & 4 & 5 & 5 & \\
\hline \multirow[t]{2}{*}{ Weight loss } & $Y(17)$ & 1 & 2 & 2 & 0.857 \\
\hline & $N(52)$ & 5 & 7 & 7 & \\
\hline \multirow[t]{2}{*}{ Night Sweat } & $Y(12)$ & 3 & 4 & 4 & $0.022^{*}$ \\
\hline & $N(57)$ & 3 & 5 & 5 & \\
\hline \multirow[t]{2}{*}{ Chest Pain } & $Y(22)$ & 3 & 4 & 4 & 0.386 \\
\hline & $N(47)$ & 3 & 5 & 5 & \\
\hline \multirow{2}{*}{$\begin{array}{l}\text { Shortness of } \\
\text { breath }\end{array}$} & $Y(7)$ & 1 & 2 & 2 & 0.198 \\
\hline & $N(62)$ & 5 & 7 & 7 & \\
\hline \multirow[t]{2}{*}{ Fatigability } & $Y(25)$ & 3 & 3 & 3 & 0.846 \\
\hline & $N(44)$ & 3 & 6 & 6 & \\
\hline \multirow[t]{2}{*}{ Symptomatic } & $Y(60)$ & 6 & 9 & 9 & 0.474 \\
\hline & $N(9)$ & 0 & 0 & 0 & \\
\hline \multirow[t]{2}{*}{ Consolidation } & $Y(7)$ & 4 & 5 & 5 & $<0.000 * *$ \\
\hline & $N(62)$ & 2 & 4 & 4 & \\
\hline \multirow[t]{2}{*}{ Cavities } & $Y(8)$ & 4 & 5 & 5 & $<0.000 * *$ \\
\hline & $N(61)$ & 2 & 4 & 4 & \\
\hline \multirow[t]{2}{*}{ Lymphadenopathy } & $Y(5)$ & 0 & 0 & 0 & 0.369 \\
\hline & $N(64)$ & 6 & 9 & 9 & \\
\hline \multirow[t]{2}{*}{ Pleural effusion } & $Y(0)$ & 0 & 0 & 0 & - \\
\hline & $N(69)$ & 6 & 9 & 9 & \\
\hline
\end{tabular}

*Significant at 0.05; **Significant at 0.01; Associations were only between TB features of HIV positive patients and positive results by the ExpertMTB/RIF test

In this study the XpertMTB/RIF correctly detected 9 patients as TB positives and 60 as non-TB cases. On the other hand, microscopy detected 5 patients who were true positives, 1 false positive, 59 true negative and 4 false negative (Table 3). Among the 9 patients with TB diagnosis none had rifampicin resistance or multi-drug resistant TB. 
Table 3: General performance results for XpertMTB/RIF and microscopy versus Lowenstein-Jensen culture

\begin{tabular}{lllll}
\hline Test & LJ positive & L negative & Total \\
\hline Microscopy negative & 5 & 1 & 6 \\
Microscopy positive & 4 & 59 & 63 \\
& Total & 9 & 60 & 69 \\
\hline Xpert positive & 9 & 0 & 9 \\
Xpert negative & 0 & 60 & 60 \\
& Total & 9 & 60 & 69 \\
\hline
\end{tabular}

Key: LJ- Lowenstein-Jensen; Microscopy sensitivity=5/9 (55.6\%); XpertMTB/RIF sensitivity=9/9 (100.0\%)

In this study, the XpertMTB/RIF had a very high sensitivity and specificity of $100 \%$ than microscopy. In addition, the test had high positive Predictive Value (PPV) of $79.2 \%$ and a Negative Predictive Value (NPV) of 98.9\%. Among those who were symptomatic for TB, XpertMTB/RIF had a similar sensitivity and specificity as the total study participants (Table 4). Sensitivity and PPV of the XpertMTB/RIF could not be calculated for the asymptomatic patients because none was found to be $L J$ culture positive.

Table 4: Comparison of XpertMTB/RIF and microscopy as compared to $\mathrm{LJ}$ as a gold standard

\begin{tabular}{lllll}
\hline $\begin{array}{l}\text { Performance } \\
\text { parameter }\end{array}$ & Test & $\begin{array}{l}\text { Performance value } \\
(\mathbf{C I})\end{array}$ & $\begin{array}{l}\text { TB clinical features } \\
\text { Symptomatic } \\
(\mathbf{n}=60)\end{array}$ & Asymptomatic $(\mathbf{n}=9)$ \\
\hline Sensitivity & XpertMTB/RIF & $100(66.4-100.0)$ & $100(66.3-100.0)$ & - \\
& Microscopy & $55.6(21.4-86.3)$ & $55.6(21.2-86.3)$ & - \\
Specificity & XpertMTB/RIF & $100(94.0-100.0)$ & $100(93.0-100.0)$ & $100(66.2-100.0)$ \\
& Microscopy & $98.3(91.0-100.0)$ & $98.3(89.6-100.0)$ & $100(66.2-100.0)$ \\
PPV & XpertMTB/RIF & $79.2(39.0-91.5)$ & $76.5(35.6-90.2)$ & - \\
& Microscopy & $75.6(28.9-95 \cdot 9)$ & $72.4(25.8-95.2)$ & - \\
NPV & XpertMTB/RIF & $98.9(95.0-99.6)$ & $98.9(95.0-99.6)$ & $100(66.2-100.0)$ \\
& Microscopy & $96.0(92.0-98.0)$ & $95.6(92.0-98.0)$ & $100(66.2-100.0)$ \\
LR (+) & XpertMTB/RIF & - & - & - \\
& Microscopy & $33.3(4.4-253.7)$ & $28.3(3.7-215.0)$ & - \\
LR (-) & XpertMTB/RIF & 0 & 0 & 1 \\
& Microscopy & $0.45(0.22-0.94)$ & $0.45(0.22-0.94)$ & 1 \\
\hline
\end{tabular}

PPV=Positive Predictive Value; NPV=Negative Predictive Value; LR (+)=Positive Likelihood Ratio; LR (-)= Negative Likelihood Ratio

Microscopy, on the other hand, had a sensitivity of $55.6 \%$, specificity of $98.3 \%$, Positive Predictive Value (PPV) of $75.6 \%$ and a Negative Predictive Value (NPV) of $96.0 \%$. Among the symptomatic patients, XpertMTB/RIF had a sensitivity of $55.6 \%$, specificity of $98.0 \%$, PPV of $72.4 \%$ and NPV of $95.6 \%$. For the asymptomatic patients, microscopy had a specificity of $100 \%$ and NPV of $100 \%$. Sensitivity and PPV for $\mathrm{LJ}$ culture could not be estimated as there was no asymptomatic patient who had TB by LJ culture.

We show in our results presented in Table 5 that the three diagnostic tests had strong correlations between them. XpertMTB/RIF had the strongest correlation with $L J$ and microscopy by 1.00 and 0.94 respectively $(p<0.01)$. We also analysed data to determine how well XpertMTB/Rif is correlated to haematological parameters. The XpertMTB/RIF results correlated with CD4 count results but not with other haematological parameters. In this case, positivity for XpertMTB/RIF significantly correlated with lower CD4 counts ( $p=0.013$ ). The median CD4 count among those with the XpertMTB/RIF positive

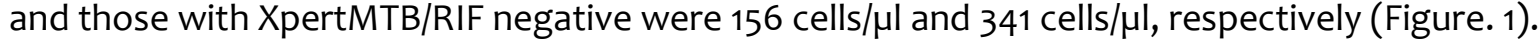


Table 5: Correlation between diagnostic tests and TB symptoms among HIV positive patients

\begin{tabular}{llllll}
\hline $\begin{array}{l}\text { Diagnostic } \\
\text { test }\end{array}$ & Symptoms & U & XpertMTB & Microscopy \\
\hline Symptoms & Correlation coefficient (CC) & 1 & $0.409^{*}$ & 0.25 & 0.25 \\
& P-value & & 0.038 & 0.218 & 0.218 \\
& Total & 26 & 26 & 26 & 26 \\
& CC & & 1 & $0.940^{* *}$ & $0.940^{* *}$ \\
& P-value & & 0 & 0 \\
XpertMTB & Total & 26 & 26 & 26 \\
& CC & & 1 & $1.000^{* *}$ \\
& P-value & & & 26 \\
Microscopy & Total & & & 26 & \\
& CC & & & \\
& P-value & & & 26 \\
& Total & & & \\
\end{tabular}

*Significant at 0.05 ; **Significant at 0.01 . XpertMTB/RIF had strong correlations with both microscopy $(C C=1.000)$ and $L J$ culture $(C C=0.940)$ *

(A) HB Vs xpert $(p=0.2005$

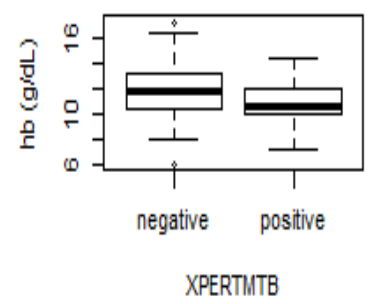

(D) GRA. Vs xpert ( $p=0.2544)$

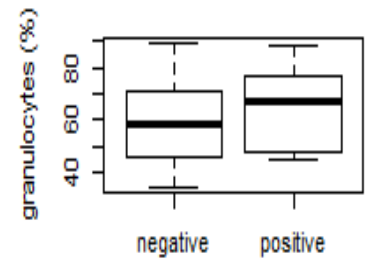

XPERTMTB
(B) LYM. Vs xpert ( $p=0.3688)$

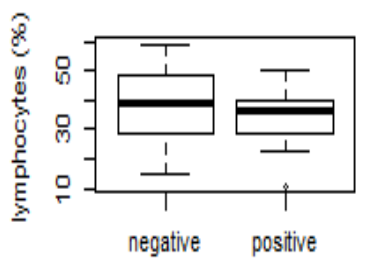

XPERTMTB

(E) MON. Vs xpert $(p=0.4284)$

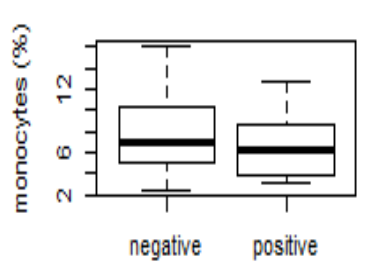

XPERTIMTB

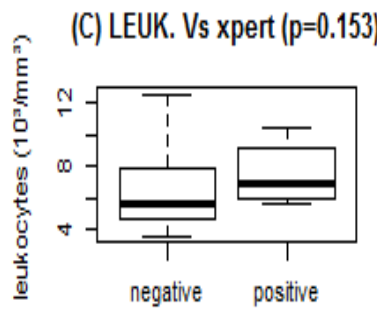

XPERTMTB

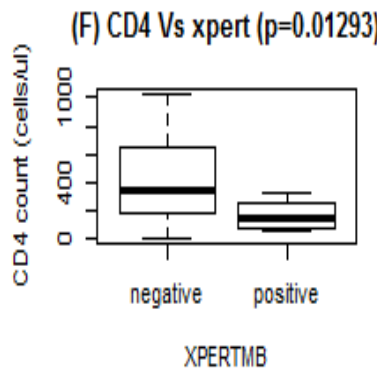

Figure 1: Correlation of XpertMTB/RIF with haematological parameters in HIV positive TB infected

Key: $h b=$ haemoglobin; LYM=lymphocytes; LEUK=leukocytes; $G R A=$ granulocytes; $M O N=$ =monocytes; $C D 4=$ cluster of differentiation 4

\section{Discussion}

The current and the previous TB diagnostic algorithms use the WHO TB symptoms screening tool as a part of the screening process. We have found that the majority of the HIV patients presented with at 
least a single TB symptom but had no TB diagnosis. This implies that using clinical TB symptoms alone as screening tool in HIV infected individuals may result into overtreatment of TB (Khan \& Starke, 1995; Siddiqi et al., 2003). The consequences for that may include misuse of anti-TB drugs and exposing the patients to unnecessary drug adverse events due to TB treatment. On the other hand, relying on microscopy alone for TB screening in HIV patients has the potential of TB under-diagnosis and missdiagnosis due to poor microscopy performance (Hawken et al., 2001) as also indicated in this study. This is because microscopy detection threshold is higher; requiring at least 150 bacilli/ $\mathrm{mL}$ of sputum which is uncommon among TB infected HIV patients. Our study has found that microscopy combined with the WHO symptoms screening tool has an inferior performance, compared to XpertMTB/RIF combined with the WHO symptoms screening tool.

None of the asymptomatic patients had TB. This finding is contrary to the report by Mtei et al. (2005) who reported about one-third of patients diagnosed with TB were asymptomatic. The reason for this difference could be explained by the observation that, most of the participants in our study had used some antibiotics at least within two weeks prior to enrolment into the study, with majority of them on CPT. Moreover, no sputum induction was attempted in our study.

Night sweat was the only symptom predictive of TB diagnosis in our current study. In a study in Vietnam, symptom screening tool had low TB predictive capacity and led to missing of about half of the smear negative HIV PTB cases (Nguyen et al., 2012). This is also an indicator that modern tests like XpertMTB/RIF with much higher accuracy in diagnosing TB are highly needed in this population. Wide variations have been observed on chest radiography among HIV patients. A study in Senegal has reported that such patients present with atypical chest radiographs, and could also present with normal or minimal chest lesions (Aderaye et al., 2004). The variability could be due to differing CD4 levels among these HIV TB co-infected patients. However, another study in Iran has reported contrary observations; that these patients' radiographic presentations are not associated with CD4 count (Bakhshayesh-Karam et al., 2004). These radiographic differences are likely to be due to variability in radiographic reading and interpretations (Balabanova et al., 2005).

Low CD4 count was positively associated with the TB diagnosis. Other studies have found a positive association between positive TB diagnosis among HIV patients and a low CD4 count of less than $200 / \mathrm{mm}^{3}$ (Nguyen et al., 2012). This could explain the fact that most of these HIV patients have an increased risk of developing TB 20-30 times compared to the general population when their CD4 count is much less (Kwan \& Ernst, 2011). This study has found that a subset of HIV patients who have lower CD4 count were likely to turn positive with XpertMTB/RIF.

In this study, the XpertMTB/RIF test showed high specificity and sensitivity. A systematic review, which had included about 27 studies about XpertMTB/RIF performance, found that XpertMTB/RIF had a pooled sensitivity of $79 \%$ and a pooled specificity of $92 \%-100 \%$ among HIV patients (Steingart et al., 2014). In this study, microscopy had sensitivity and a specificity of $55.6 \%$ and $98.3 \%$, respectively which implies lower performance compared to XpertMTB/RIF. The high sensitivity of XpertMTB/RIF implies that an additional $44.4 \%$ of HIV positive TB infected individuals who would otherwise be missed by microscopy are diagnosed by XpertMTB/RIF test. Similar microscopy performances when compared to XpertMTB/RIF have been reported in previous studies (Boehme et al., 2010; Theron et al., 2011; Ntinginya et al., 2012; Opota et al., 2016).

The probability of having the disease when one tests positive and the probability of not having the disease when one tests negative seems to be slightly higher for XpertMTB/RIF as compared to microscopy. Similar findings have been observed among the symptomatic patients (Ioannidis et al., 2011; Lawn et al., 2011, Opota et al., 2016). For the asymptomatic patients a negative test, from any of the two tests (XpertMTB/RIF or microscopy), seems to exclude the disease, as NPV was 100\% (95\% CI $66.2 \%-100 \%)$. This could be attributed to the relatively limited small sample size in our study because some of TB patients might have been asymptomatic as previously reported (Mtei et al., 2005). In this 
aspect, the performance of XpertMTB/RIF was not significantly different from that of microscopy. This was due to the broad confidence intervals resulting from the small sample size in our study. Despite this limitation, our results indicate that XpertMTB/RIF is highly sensitive and specific to detect all culture positive TB cases among HIV patients. We recommend the adoption of XpertMTB/RIF as an early TB diagnosis tool among HIV patients for early detection of TB among HIV patients.

\section{Conflicts of interests}

Authors declare no conflict of interests.

\section{Acknowledgements}

We are grateful to all study participants who made this work possible. Staffs of Kilimanjaro Christian Medical University College, Kibong'oto Infectious Diseases Hospital and Kilimanjaro Clinical Research Institute are thanked for their constructive ideas, work and time in this research project. We acknowledge the Government of Tanzania through the Ministry of Health and Social Welfare for funding this study.

\section{References}

Aderaye, G., Bruchfeld, J., Assefa, G., Feleke, D., Källenius, G., Baat, M. \& Lindquist, L. (2004) The relationship between disease pattern and disease burden by chest radiography, $M$. tuberculosis load, and HIV status in patients with pulmonary tuberculosis in Addis Ababa. Infection 32, 333-338.

Bakhshayesh-Karam, M., Tabarsi, P., Mirsaiedi, S.M., Amiri, M.V., Zahirifard, S., Mansoori, S.D., Masjedi, M.R. \& Velayati, A.A. (2004) Radiographic manifestations in TB/HIV Patients. Tanaffos 3, 33-39.

Balabanova,Y., Coker, R., Fedorin, I., Zakharova, S., Plavinskij, S., Krukov, N., Atun, R. \& Drobniewski, F. (2005) Variability in interpretation of chest radiographs among Russian clinicians and implications for screening programmes: observational study. BMJ 331, 379-382.

Balcells,M.E., Thomas,S.L., Godfrey-Faussett,P., \& Grant,A.D. (2006) Isoniazid preventive therapy and risk for resistant tuberculosis. Emerging infectious diseases 12, 744-751.

Boehme, C.C., Nabeta, P., Hillemann, D., Nicol, M.P., Shenai, S., Krapp, F., Allen, J., Tahirli, R., Blakemore, R. \& Rustomjee, R. (2010) Rapid molecular detection of tuberculosis and rifampin resistance. New England Journal of Medicine 363, 1005-1015.

Gandhi, N.R., Shah, N.S., Andrews, J.R., Vella, V., Moll, A.P., Scott, M., Weissman, D., Marra, C., Lalloo, U.G. \& Friedland, G.H. (2010) HIV coinfection in multidrug-and extensively drug-resistant tuberculosis results in high early mortality. American Journal of Respiratory and Critical Care Medicine 181, 80-86.

Hawken, M.P., Muhindi, D.W., Chakaya, J.M., Bhatt, S.M., Ng'ang'a, L.W. \& Porter, J.D.H. (2001) Underdiagnosis of smear-positive pulmonary tuberculosis in Nairobi, Kenya. The International Journal of Tuberculosis and Lung Disease 5, 360-363.

Ioannidis, P., Papaventsis, D., Karabela, S., Nikolaou, S., Panagi, M., Raftopoulou, E., Konstantinidou, E., Marinou, I. \& Kanavaki, S. (2011) Cepheid GeneXpert MTB/RIF assay for Mycobacterium tuberculosis detection and rifampin resistance identification in patients with substantial clinical indications of tuberculosis and smear-negative microscopy results. Journal of Clinical Microbiology 49, 3068-3070.

Khan, E.A. \& Starke, J.R. (1995) Diagnosis of tuberculosis in children: increased need for better methods. Emerging Infectious Diseases 1, 115. 
Kwan, C.K. \& Ernst, J.D. (2011) HIV and tuberculosis: a deadly human syndemic. Clinical Microbiology Reviews 24, 351-376.

Lawn, S.D., Brooks, S.V., Kranzer, K., Nicol, M.P., Whitelaw, A., Vogt, M., Bekker, L.G. \& Wood, R. (2011) Screening for HIV-associated tuberculosis and rifampicin resistance before antiretroviral therapy using the Xpert MTB/RIF assay: a prospective study. PLoS Medicine 8, e1001067.

Mtei, L., Matee, M., Herfort, O., Bakari, M., Horsburgh, C.R., Waddell, R., Cole, B.F., Vuola, J.M., Tvaroha, S. \& Kreiswirth, B. (2005) High rates of clinical and subclinical tuberculosis among HIV-infected ambulatory subjects in Tanzania. Clinical Infectious Diseases 40, 1500-1507.

Ngowi, B.J., Mfinanga, S.G., Bruun, J.N. \& Morkve,O. (2008) Pulmonary tuberculosis among people living with HIV/AIDS attending care and treatment in rural northern Tanzania. BMC Public Health $8,1$.

Nguyen, D., Nguyen, H.Q., Beasley, R.P., Ford, C.E., Hwang, L.Y. \& Graviss, E.A. (2012) Performance of clinical algorithms for smear-negative tuberculosis in HIV-infected persons in Ho Chi Minh City, Vietnam. Tuberculosis Research and Treatment 2012, Article ID 360852, http://dx.doi.org/10.1155/2012/360852

Ntinginya, E.N., Squire, S.B., Millington, K.A., Mtafya, B., Saathoff, E., Heinrich, N., Rojas-Ponce, G., Kowuor, D., Maboko, L. \& Reither, K. (2012) Performance of the Xpert ${ }^{\circledR}$ MTB/RIF assay in an active case-finding strategy: a pilot study from Tanzania [Short communication]. The International Journal of Tuberculosis and Lung Disease 16, 1468-1470.

Opota, O., Senn, L., Prod'hom, G., Mazza-Stalder, J., Tissot, F., Greub, G. \& Jaton, K. (2016) Added value of the molecular assay Xpert MTB/RIF compared to sputum smear microscopy to assess the risk of tuberculosis transmission in a low-prevalence country. Clinical Microbiology and Infection 22, 613-619.

Siddiqi, K., Lambert, M.L. \& Walley, J. (2003) Clinical diagnosis of smear-negative pulmonary tuberculosis in low-income countries: the current evidence. Lancet Infectious Diseases 3, 288296.

Steingart, K.R., Schiller, I., Horne, D.J., Pai, M., Boehme, C.C. \& Dendukuri, N. (2014) Xpert ®-MTB/RIF assay for pulmonary tuberculosis and rifampicin resistance in adults. Cochrane Database Systematic Review 21(1):CDo09593. doi: 10.1002/14651858.CDo09593.pub3.

Swai, H.F., Mugusi, F.M. \& Mbwambo, J.K. (2011) Sputum smear negative pulmonary tuberculosis: sensitivity and specificity of diagnostic algorithm. BMC Research Notes 4, 475.

Theron, G., Peter, J., van Zyl-Smit, R., Mishra, H., Streicher, E., Murray, S., Dawson, R., Whitelaw, A., Hoelscher, M. \& Sharma, S. (2011) Evaluation of the Xpert MTB/RIF assay for the diagnosis of pulmonary tuberculosis in a high HIV prevalence setting. American Journal of Respiratory and Critical Care Medicine 184, 132-140.

WHO (2009) Global Tuberculosis Control: a Short Update to the 2009 Report. World Health Organization, Geneva, Switzerland.

WHO (2013) Global Tuberculosis Control 2011. World Health Organization, Geneva, Switzerland. WHO (2015) Global Tuberculosis Report 2015. World Health Organization, Geneva, Switzerland. 